\title{
COMMUTATIVE RINGS CONTAINING AT MOST TWO PRIME IDEALS
}

\author{
Robert W. Gilmer
}

\section{INTRODUCTION}

This paper considers the structure and ideal theory of a commutative ring $R$ with at most two prime ideals. If $\mathrm{R}$ contains a unit element, then $\mathrm{R}$ is a primary ring in the sense of Zariski-Samuel [3, p. 204]. The following definition is therefore a generalization of that of Zariski-Samuel: A primary ring is a commutative ring containing at most two prime ideals. A primary domain is a primary ring containing no proper zero divisors. As already noted, the only case of interest is in the study of primary rings without unit.

If $A$ is an ideal of a commutative ring $R$, then $\sqrt{A}$ is the intersection of all prime ideals of $R$ containing $A[1, p .9]$. If $R$ is a primary ring with prime ideals $P$ and $R$, this means $\sqrt{A}=P$ or $\sqrt{A}=R$. This simple observation serves as a starting point for our investigations. In fact, the commutative ring $R$ is a primary ring if and only if given $b \in R, b$ is nilpotent or $\sqrt{(b)}=R$. If $S$ is a ring in which $\sqrt{(0)}=\mathrm{P}$ is a proper prime ideal, if $\mathrm{M}$ is the intersection of all prime ideals of $\mathrm{S}$ properly containing $P$, and if $Q$ is an ideal of $S$ contained in $M$, then $Q$ is a primary ring. For a primary domain $D$ we can state, conversely: there exists a domain $J$ (hence $\sqrt{(0)}=(0)$ is prime) with unit such that $D$ is an ideal of $J$ contained in the intersection of all non-zero prime ideals of $\mathrm{J}$. One can state much stronger results of a similar nature if $S$ or $D$ is Noetherian-for example, if $D$ is a Noetherian primary domain, then there exists a semi-local domain $J$ with unit such that $D$ is an ideal of $\mathrm{J}$ contained in the Jacobson radical of $\mathrm{J}$. An interesting theorem is proved in connection with the converse of the previous result; namely, that an ideal $Q$ contained in the Jacobson radical of a semi-local domain $D$ with unit is itself a Noetherian ring if and only if each maximal ideal of $D$ has finite index in $D$ when considered as a subgroup of the additive group of $D$.

All rings considered in this paper will be assumed to be commutative and to contain more than one element. If $\mathrm{D}$ is an integral domain, $\mathrm{Q}(\mathrm{D})$ will denote the quotient field of $D$ and $D^{*}$ will denote the subring of $Q(D)$ generated by $D$ and the identity element of $\mathrm{Q}(\mathrm{D})$. $\mathrm{Z}$ will always denote the ring of integers. The terminology is that of Zariski-Samuel [3] [4].

\section{PRIMARY RINGS}

In riew of the remarks made previously, all primary rings considered in the remainder of this paper will be assumed to contain no unit element. There are essentially two cases to consider then in examining a primary ring $R$. One is the case where $R$ contains no proper prime ideals. This occurs if and only if every element of $R$ is nilpotent. The more interesting case is where $R$ contains a proper prime ideal $P$. In this case $\sqrt{(0)}=P$ and if $b \in R-P, \sqrt{(b)}=R$. That these two properties determine a primary ring is shown by

Received November 6, 1962. 
THEOREM 1. A ring $\mathrm{R}$ is a primary ring if given $\mathrm{b} \in \mathrm{R}$, either $\mathrm{b}$ is nilpotent or $\sqrt{(\mathrm{b})}=\mathrm{R}$.

Proof. If $\mathrm{P}$ is a prime ideal of $\mathrm{R}, \mathrm{P}$ contains every nilpotent element of $\mathrm{R}$. Therefore either $P$ is the set of nilpotent elements or $P=R$. Hence $R$ contains at most two prime ideals.

COROLLARY 1. The integral domain $\mathrm{D}$ is a primary domain if and only if given $\mathrm{a}, \mathrm{b} \in \mathrm{D}$ with $\mathrm{b} \neq 0$, there exist $a \mathrm{k} \in \mathrm{Z}$ and $a \mathrm{~d} \in \mathrm{D}$ such that $\mathrm{a} \mathrm{k}=\mathrm{db}$.

Proof. That the condition is sufficient follows from Theorem 1. If $\mathrm{D}$ is a primary domain, then $b^{2} \neq 0$ implies $\sqrt{\left(b^{2}\right)}=D[1, p .9]$ so that for some $t \in D$ and $\mathrm{n}, \mathrm{k} \in \mathrm{Z}, \mathrm{a}^{\mathrm{k}}=\mathrm{tb}^{2}+\mathrm{nb}^{2}=(\mathrm{tb}+\mathrm{nb}) \mathrm{b}=\mathrm{db}$.

THEOREM 2. A ring $\mathrm{R}$ is a primary ring if and only if $\sqrt{(0)}=\mathrm{P}$ is a prime ideal and $\mathrm{R} / \mathrm{P}$ is a primary domain.

Proof. A primary ring has these two properties. If a ring $\mathrm{R}$ has these two properties and $P_{1}$ is a prime ideal of $R, P_{1} \supseteq P$. If $P_{1} \supset P$, then $P_{1} / P$ is a nonzero prime ideal of $R / P$ so that $P_{1} / P=R / P, P_{1}=R$, and the result follows.

We now consider the problem of construction of primary rings. We first show how any primary domain may be obtained. The previous simple results lead to the following.

THEOREM 3. If $\mathrm{J}$ is an integral domain, if $\mathrm{M}$ is the intersection of all nonzero prime ideals of $\mathrm{J}$, and if $\mathrm{D}$ is an ideal of $\mathrm{J}$ contained in $\mathrm{M}$, then $\mathrm{D}$ is a primary domain. Conversely, if $\mathrm{D}_{0}$ is a primary domain, then there exists an integral domain $\mathrm{J}_{0}$ with unit such that $\mathrm{D}_{0}$ is an ideal of $\mathrm{J}_{0}$ contained in the intersection of all non-zero prime ideals of $\mathrm{J}_{0}$.

The following lemma will be needed in the proof of the second part of Theorem 3 and later on in this paper.

LEMMA 1. Let $\mathrm{S}$ be a ring with unit $\mathrm{e}$, and let $\mathrm{R}$ be a subring of $\mathrm{S}$ such that $\mathrm{S}$ is generated by $\mathrm{R}$ and e. A subset of $\mathrm{R}$ is an ideal of $\mathrm{S}$ if and only if it is an ideal of $\mathrm{R}$. $\mathrm{S}$ is Noetherian if and only if $\mathrm{R}$ is Noetherian. Finally, if $\mathrm{S}$ is a domain, if $\mathrm{M}$ is the intersection of all non-zero prime ideals of $\mathrm{S}$, and if $\mathrm{N}$ is the intersection of all non-zero prime ideals of $\mathrm{R}$, then $\mathrm{N} \subseteq \mathrm{M}$.

Proof. Clearly an ideal of $\mathrm{S}$ contained in $\mathrm{R}$ is an ideal of $\mathrm{R}$. If $\mathrm{A}$ is an ideal of $R$, a $\epsilon \mathrm{A}$, and $\mathrm{d}=\mathrm{r}+\mathrm{ne} \epsilon \mathrm{S}$, then $\mathrm{da}=\mathrm{ra}+\mathrm{na} \epsilon \mathrm{A}$ also. Hence $\mathrm{A}$ is an ideal of S.

If $S$ is Noetherian, $R$ is Noetherian by what was just proved. To prove the converse we take an ideal $A$ of $S$ and show $A$ has a finite basis. Thus $A_{1}=A \cap R$ is an ideal of $R$, and we may choose a finite basis $\left\{a_{1}, \cdots, a_{s}\right\}$ of $A_{1}$ in $R$ if $R$ is Noetherian. If $B$ is the collection of all integers $n$ such that $r+n e \in A$ for some $r \in R$, then $B$ is an ideal of $Z$ and therefore is generated by some single element $b$. If $a=x+b e \in A$ and if $a^{*}=y+m e \in A$, then for some integer $q$, $a^{*}-q a \in A_{l}$. It follows that $\left\{a_{1}, \cdots, a_{s}, a\right\}$ is a basis of $A$ in $S$.

If $P$ is a non-zero prime ideal of $S$ and $S$ is a domain, then $(0) \subset R P \subset R \cap P$ so that $R \cap P$ is a non-zero prime ideal of $R$. It follows that $M \supseteq N$.

Proof of Theorem 3. We first show $D$ is a primary domain. Thus if $a, b \in D$ and $b \neq 0$, we denote by $(b)_{J}$ and $(b)_{D}$ the principal ideals generated by $b$ in $J$ and $D$, respectively. $\sqrt{(b)_{J}}$ is the intersection of all prime ideals of $J$ containing $b$. Hence $\mathrm{D} \subseteq \mathrm{M} \subseteq \sqrt{(\mathrm{b})} \mathrm{J}$. It follows that for some $\mathrm{k}, \mathrm{n} \in \mathrm{Z}$ and some $\mathrm{d} \in \mathrm{J}$, $a^{k}=d b+\bar{n} b$. Consequently $a^{k+1}=(d a+n a) b$ and $d a+n a \in D$. By Corollary $1, D$ is a primary domain. 
Next, we suppose $D_{0}$ is a primary domain and we let $J_{0}=D_{0} * \cdot J_{0}$ is an integral domain with unit, and Lemma 1 shows $D_{0}$ is an ideal of $J_{0}$ contained in every non-zero prime ideal of $\mathrm{J}_{0}$.

COROLLARY 2. If $\mathrm{S}$ is a ring in which $\sqrt{(0)}=\mathrm{P}$ is a proper prime ideal, if $\mathrm{M}$ is the intersection of all prime ideals of $\mathrm{S}$ properly containing $\mathrm{P}$, and if $\mathrm{Q}$ is an ideal of $\mathrm{S}$ contained in $\mathrm{M}$, then $\mathrm{Q}$ is a primary ring.

Proof. We note that $\mathrm{P} \cap \mathrm{Q}$ is the set of nilpotent elements of $\mathrm{Q}$. Now $\mathrm{Q} / \mathrm{P} \cap \mathrm{Q} \simeq \mathbf{P}+\mathrm{Q} / \mathrm{P} \subseteq \mathrm{M} / \mathrm{P}$ - which, by Theorem 3 as applied to the domain $\mathrm{S} / \mathrm{P}$, is a primary domain. Corollary 2 now follows from Theorem 2 in view of the following lemma.

\section{LEMMA 2. If $\mathrm{A}$ is an ideal of the primary ring $\mathrm{R}$, then $\mathrm{A}$ is a primary ring.}

Proof. Suppose $\mathrm{x} \in \mathrm{A}$ and $\mathrm{x}$ is not nilpotent. Then the principal ideal generated by $x$ in $R$ has radical $R$ by Theorem 1. If then $a \in A$, we see that $a^{k}=v x+n x$ for some $v \in R$ and some $n, k \in Z$. Hence $a^{k+1}=(v a+n a) x$ and $v a+n a \epsilon A$ since $A$ is an ideal of $R$. Theorem 1 now implies Lemma 2 .

In view of Corollary 2 and Theorem 3, we might expect the following statement to be true: If $R_{0}$ is a primary ring, then there exists a ring $S_{0}$ with unit in which $\sqrt{(0)}=P_{0}$ is a proper prime ideal and such that $R_{0}$ is an ideal of $S_{0}$ contained in the intersection of all prime ideals of $S_{0}$ which properly contain $P_{0}$. That this statement is false is shown by the following example. We let

$$
\mathrm{R}_{0}=\left\{2^{*}, 4^{*}, 6^{*}, 8^{*}, 10^{*}, 0^{*}\right\}
$$

denote the ring of residue classes of the ring of even integers modulo the principal ideal generated by 12 . $R_{0}$ is a primary ring. Yet in any ring $S_{0}$ with unit e containing $R_{0}$, the set of nilpotent elements will not form a prime ideal. This is true because $\left(2^{*}\right)\left(2^{*}+\mathrm{e}\right)$ is nilpotent, but neither $2^{*}$ nor $2^{*}+\mathrm{e}$ can be. For $2^{*}$ this is obvious. Any power of $2^{*}+\mathrm{e}$ may be written in the form $r+e$ for some $r \in R$. Since $\mathrm{e} \notin \mathrm{R}$, no power of $2^{*}+\mathrm{e}$ is zero.

Remark. It can be seen that if the requirement that $\mathrm{S}_{0}$ have a unit is dropped, then the resulting statement is true. The author has been unable to find an exact analog to Theorem 3 in the case of primary rings. If we take $S_{0}=Z /(12)$ and $R_{0}$ as in the previous example, the difficulty of describing $R_{0}$ in terms of the prime ideals of $S_{0}$ is illustrated, even in the case when $R_{0}$ and $S_{0}$ are Noetherian.

\section{NOETHERIAN PRIMARY RINGS}

As we might expect, the results of the previous section may be strengthened if we consider rings which are Noetherian. Before we begin, we note that the primary domain constructed in Theorem 3 is trivial if $M=(0)$. Similarly, in Corollary $2, \mathbf{Q}$ is a "trivial" primary ring in the sense that every element of $Q$ is nilpotent if $M=P$. Accordingly, we shall be interested in analogs to Theorem 3 and Corollary 2 in the cases where $M \neq(0)$ and $M \supset P$, respectively.

Definition. The Jacobson radical of a ring $\mathrm{R}$ with unit is the intersection of all maximal ideals of $\mathrm{R}$.

THEOREM 4. If $\mathrm{J}$ is a Noetherian domain with unit, not a field, such that the intersection $\mathrm{M}$ of all non-zero prime ideals of $\mathrm{J}$ is non-zero, then $\mathrm{J}$ is a onedimensional (see [2]) semi-local ring and $\mathrm{M}$ is the Jacobson radical of $\mathrm{J}$. 
Proof. We let $\mathrm{m}$ be a non-zero element of $\mathrm{M}$ and we let

$$
(\mathrm{m})=\mathrm{Q}_{1} \cap \cdots \cap \mathrm{Q}_{\mathrm{k}}
$$

be an irredundant representation of $(m)$ in $J$, where $Q_{i}$ is $P_{i}$-primary. Suppose $P_{1}, \cdots, P_{t}$ are the minimal primes of $(m)$. If $P$ is any non-zero proper prime ideal of $\mathrm{J}$, then $\mathrm{m} \in \mathrm{P}$ implies some $\mathrm{P}_{\mathrm{i}} \subseteq \mathrm{P}(1 \leq \mathrm{i} \leq \mathrm{t})$. Hence $\mathrm{P}_{1}, \cdots, \mathrm{P}_{\mathrm{t}}$ are all the minimal prime ideals of $J$. Now if $p \in P$, then the principal ideal theorem [3, $p$. 238] implies that $p$ is in some minimal prime ideal of J. It follows that

$$
\mathrm{P} \subseteq \bigcup_{i=1}^{\mathrm{t}} \mathrm{P}_{\mathrm{i}}
$$

so that $P=P_{i}$ for some $i[3, p .215]$. Hence $P_{1}, \cdots, P_{t}$ are all the non-zero proper prime ideals of $\mathrm{J}$, and each of these is minimal. The conclusions of Theorem 4 then follow.

COROLLARY 3. If $\mathrm{R}$ is a Noetherian ring with unit such that $\sqrt{(0)}=\mathbf{P}$ is a nonmaximal proper prime ideal of $\mathrm{R}$, and if the intersection $\mathrm{M}$ of all prime ideals of $\mathrm{R}$ properly containing $\mathrm{P}$ is not $\mathrm{P}$, then $\mathrm{R}$ is one-dimensional and $\mathrm{M}$ is the Jacobson radical of $\mathrm{R}$.

Proof. The corollary follows immediately by application of Theorem 4 to the domain $\mathrm{R} / \mathrm{P}$.

Remark. Lemma 1 shows that there is no loss of generality in Theorem 4 by assuming that $\mathrm{J}$ has a unit.

THEOREM 5. Let $\mathrm{R}$ be a Noetherian primary ring such that $\sqrt{(0)}=\mathrm{P} \subset \mathrm{R}$. Let $\mathrm{R}^{*}$ be a ring with unit $\mathrm{e}$ containing $\mathrm{R}$ such that $\mathrm{R}^{*}$ is generated by $\mathrm{R}$ and $\mathrm{e}$. Then $\mathrm{R}^{*}$ contains only a finite number of prime ideals and every proper prime ideal of $\mathrm{R}^{*}$ is maximal or minimal.

Proof. By Lemma $1, \mathrm{R}^{*}$ is a Noetherian ring. If $\mathrm{P}^{*}$ is prime in $\mathrm{R}^{*}$, either $\mathrm{P}^{*} \cap \mathrm{R}=\mathrm{R}$ or $\mathrm{P}^{*} \cap \mathrm{R}=\mathrm{P}$. We shall show that if $\mathrm{P}_{1}^{*}$ and $\mathrm{P}_{2}^{*}$ are prime in $\mathrm{R}^{*}$, if $\mathrm{P}_{1}^{*} \cap \mathrm{R}=\mathrm{P}$, and if $\mathrm{P}_{1}^{*} \subset \mathrm{P}_{2}^{*}$, then $\mathrm{P}_{2}^{*} \cap \mathrm{R}=\mathrm{R}$. Thus if

$$
\mathrm{d}=\mathrm{r}+\text { ne } \epsilon \mathrm{P}_{2}^{*}-\mathrm{P}_{1}^{*}
$$

and if $b \in R-P$, then $b d \in P_{2}^{*} \cap R$, bd $\notin P_{1}^{*}$. Therefore $P_{2}^{*} \cap R=R$ as asserted. This implies that if $\mathrm{P}^{*} \cap \mathrm{R}=\mathrm{P}, \mathrm{P}^{*}$ is a minimal prime ideal of $\mathrm{R}^{*}$. We let $\mathfrak{S}=\left\{P_{1}^{*}, \cdots, P_{k}^{*}\right\}$ be the set of prime ideals of $R^{*}$ that intersect $R$ in $P$. The set $\subseteq$ is non-empty for if $P=Q_{1} \cap \cdots \cap Q_{a}$ is an irredundant representation of the ideal $P$ in $R^{*}$, where $Q_{i}$ is $P_{i}$-primary, then $\sqrt{P}=P_{1} \cap \ldots \cap P_{a}$. Now $\sqrt{P} \cap R=P$ since $P$ is prime in $R$. Therefore for some $j$ with $1 \leq j \leq a, P_{j} \pm R$ so that $P_{j} \in \mathfrak{S}$. Now for $1 \leq i \leq k, R^{*} / P_{i}^{*}$ is a Noetherian domain with unit such that the intersection of all non-zero prime ideals of $R^{*} / P_{i}^{*}$ contains $R+P_{i}^{*} / P_{i}^{*} \neq P_{i}^{*} / P_{i}^{*}$. By Theorem $4, \mathrm{R}^{*} / \mathrm{P}_{\mathrm{i}}^{*}$ is a one-dimensional semi-local ring. The proof follows immediately from this observation.

COROLLARY 4. If $\mathrm{D}_{0}$ is a Noetherian primary domain, then $\mathrm{D}_{0}^{*}$ is a onedimensional semi-local ring and $\mathrm{D}_{0}$ is an ideal of $\mathrm{D}_{0}^{*}$ contained in the Jacobson radical of $\mathrm{D}_{0}^{*}$. If $\mathrm{D}_{0}^{*}$ has characteristic $\mathrm{p} \neq 0$, then $\mathrm{D}_{0}^{*}$ is a local ring and $\mathrm{D}_{0}$ is its unique maximal ideal. (Note that $\mathrm{D}_{0}, \mathrm{D}_{0}^{*}$, and $\mathrm{Q}\left(\mathrm{D}_{0}\right)=\mathrm{Q}\left(\mathrm{D}_{0}^{*}\right)$ all have the same characteristic.) If $\mathrm{D}_{0}^{*}$ has characteristic 0 , then $\mathrm{D}_{0}^{*} / \mathrm{D}_{0} \simeq \mathrm{Z} /(\mathrm{s})$ for some integer 
$\mathrm{s}>0$. The maximal ideals of $\mathrm{D}_{0}^{*}$ in this case are $\mathrm{D}_{0}+\left(\mathrm{p}_{1} \mathrm{e}\right), \cdots, \mathrm{D}_{0}+\left(\mathrm{p}_{\mathrm{k}} \mathrm{e}\right)$ where $\mathrm{p}_{1}, \cdots, \mathrm{p}_{\mathrm{k}}$ are the prime divisors of $\mathrm{s}$.

Proof. Theorem 5 and Lemma 1 imply the conclusions of the first sentence of the corollary.

If $C$ is the set of all elements ke of $D_{0}^{*}$ such that $k \in Z$, then $D_{0}^{*}=D_{0}+C$. Therefore $D_{0}^{*} / D_{0} \simeq C / D_{0} \cap C \simeq Z /(s)$ for some integer $s$. If $C$ is a finite field of $p$ elements, then $\bar{D}_{0} \cap C=(0)$ and $s=p$. Consequently, $D_{0}^{*}$ is a local ring and $D_{0}$ is its maximal ideal. If $\mathrm{C}$ is not finite, that is, if $\mathrm{D}_{0}^{*}$ has characteristic 0 , then because $D_{0}^{*}$ is a semi-local ring $D_{0}^{*} / D_{0}$, and hence $\mathrm{Z} /(\mathrm{s})$, has only finitely many prime ideals. Hence $s \neq 0$. Further $\left(p_{i}\right) /(s)(i=1,2, \cdots, k)$ are the maximal ideals of $\mathrm{Z} /(\mathrm{s})$. Therefore

$$
D_{0}+\left(p_{1} e\right), \cdots, D_{0}+\left(p_{k} e\right)
$$

are the maximal ideals of $\mathrm{D}_{0}^{*}$.

Notation. If $\mathrm{A}$ is an ideal of the ring S, then [S: A] will denote the index of A in $S$-that is, the cardinality of the set of cosets of $A$ in $S$ when $A$ is considered as a subgroup of the additive group of $S$.

The following theorem shows in what case the Jacobson radical of a onedimensional semi-local domain is itself a Noetherian domain.

THEOREM 6. Suppose $\mathrm{J}$ is a one-dimensional semi-local domain with Jacobson radical M. A non-zero ideal $\mathrm{Q}$ of $\mathrm{J}$ contained in $\mathrm{M}$ is itself a Noetherian domain if and only if [J: Q] is finite. In order that $\mathrm{Q}$ have finite index in $\mathrm{J}$, it is necessary and sufficient that $\mathrm{M}$ have finite index in J. Finally, $[\mathrm{J}: \mathrm{M}]$ is finite if and only if [J: $\mathrm{M}_{\mathrm{i}}$ ] is finite for each maximal ideal $\mathrm{M}_{\mathrm{i}}$ of $\mathrm{J}$.

Proof. If $\mathrm{M}_{1}, \cdots, \mathrm{M}_{\mathrm{t}}$ are the maximal ideals of $\mathrm{J}$, then [3, p. 178]

$$
\mathrm{J} / \mathrm{M} \simeq \mathrm{J} / \mathrm{M}_{1} \oplus \cdots \oplus \mathrm{J} / \mathrm{M}_{\mathrm{t}}
$$

Hence $[J: M]$ is finite if and only if $\left[J: M_{\mathfrak{i}}\right]$ is finite for each $i$.

We next suppose that $[\mathrm{J}: \mathrm{M}]$ is finite. Since any non-zero ideal A of $\mathrm{J}$ contains a power of $M$, to show [J: Q] is finite, it suffices to show [ $\mathrm{J}: \mathrm{M}^{\mathrm{r}}$ ] is finite for any integer $r$. This we prove by induction, the case $r=1$ being true by hypothesis. Suppose now that $\left[\mathrm{J}: \mathrm{M}^{\mathrm{s}}\right]$ is finite. $\mathrm{M}^{\mathrm{s}} / \mathrm{M}^{\mathrm{s}+1}$ is canonically a vector space over the field $J / M$. Since $J$ is Noetherian, this space is finitely generated. Because $J / M$ is finite by assumption $\left[\mathrm{M}^{\mathrm{s}}: \mathrm{M}^{\mathrm{s+1}}\right]$ is finite. Then by the induction hypothesis

$$
\left[\mathrm{J}: \mathrm{M}^{\mathrm{S}+1}\right]=\left[\mathrm{J}: \mathrm{M}^{\mathrm{S}}\right]\left[\mathrm{M}^{\mathrm{S}}: \mathrm{M}^{\mathrm{S}+1}\right]
$$

is finite. It is clear that if there exists an ideal $Q \subseteq M$ such that [ $J: Q]$ is finite, then $[\mathrm{J}: \mathrm{M}]$ is finite. We may summarize by saying: A necessary and sufficient condition that $[\mathrm{J}: \mathrm{A}]$ be finite for each non-zero ideal $A$ of $\mathrm{J}$ is that $[\mathrm{J}: \mathrm{M}]$ be finite.

Now suppose that the ideal $\mathrm{Q} \subseteq \mathrm{M}$ has finite index in J. If $\mathrm{B}$ is a non-zero ideal of the ring $\mathrm{Q}$, then $\mathrm{BQ}$ is a non-zero ideal of $\mathrm{J}$ and $\mathrm{BQ} \subseteq \mathrm{B} \subseteq \mathrm{Q} \subset \mathrm{J}$. From the preceding paragraph, $[\mathrm{J}: \mathrm{BQ}]$ is finite. Therefore $[\mathrm{Q}: \mathrm{B}]$ is finite and $\mathrm{Q}$ is a Noetherian ring.

Conversely, suppose that $Q$ is Noetherian. Let $q$ be a non-zero element of $Q$, and let $\mathrm{e}$ be the identity of $\mathrm{J}$. The ideal $\mathrm{Jq}$ of $\mathrm{J}$ is contained in $\mathrm{Q}$ and is an ideal 
of $Q$. Let $r_{1} q, \cdots, r_{t} q$ be a basis for the ideal Jq in $Q$. Then if $d \in J$, there exist elements $q_{i} \in Q$ and integers $n_{i}$ such that $d q=\Sigma\left(q_{i}+n_{i} e\right) r_{i} q$. Thus $\mathrm{d}=\Sigma\left(\mathrm{q}_{\mathrm{i}}+\mathrm{n}_{\mathrm{i}} \mathrm{e}\right) \mathrm{r}_{\mathrm{i}}$, and modulo $\mathrm{Q}, \mathrm{d} \equiv \Sigma \mathrm{n}_{\mathrm{i}} \mathbf{r}_{\mathrm{i}}$, where $\mathrm{r}_{1}, \cdots, \mathrm{r}_{\mathrm{t}}$ are fixed elements of $R$. Now $Q^{*} \subset J$ and $Q^{*}$ is a one-dimensional semi-local ring by Corollary 4 . Also by Corollary 4, there exists an integer $\mathbf{S} \neq 0$ such that se $\epsilon \mathbf{Q}$. This means that in the congruence above we may require that each $n_{\mathbf{i}}$ be such that $0 \leq n_{\mathbf{i}}<\mathrm{s}$. Therefore [J: Q] is finite as asserted.

\section{REFERENCES}

1. W. Krull, Idealtheorie, Chelsea Publ. Co., New York, 1948.

2. A. Seidenberg, A note on the dimension theory of rings, Pacific J. Math. 3 (1953), 505-512.

3. O. Zariski and P. Samuel, Commutative Algebra, Vol. 1, D. Van Nostrand Co., Inc., Princeton, 1958.

4. - Commutative Algebra, Vol. 2, D. Van Nostrand Co., Inc., Princeton, 1960.

University of Wisconsin 